\title{
INCIDENCE OF HAEMOLYTIC DISEASE OF THE NEWBORN IN THE OFFSPRING OF SISTERS
}

\author{
BY \\ J. W. GERRARD and J. A. H. WATERHOUSE
}

From the Department of Paediatrics and Child Health and the Department of Medical Statistics, University of Birmingham

Haemolytic disease of the newborn most commonly occurs when an Rh-negative mother has been sensitized to the $D$ antigen, either from an Rh-positive foetus during pregnancy, or by an injection or transfusion of blood. Sensitization, however, occurs much less commonly than is theoretically possible; and Wiener (1946) has suggested that the ability of an individual to become sensitized is conditioned by a certain constitutional factor, $K$, as well as by exposure to a foreign antigen. $\mathrm{He}$ suggests that homozygous $K K$ individuals $(0.02$ per cent. of the population) respond readily to antigenic stimuli, that heterozygous $K k$ individuals (3 per cent. of the population) are moderately easy to sensitize, and that homozygous $k k$ individuals (97 per cent. of the population) are difficult to sensitize. If such a constitutional factor is operative, haemolytic disease of the newborn might be expected to arise with greater frequency in the offspring of women whose sisters had had children with haemolytic disease.

The measure of this effect may be computed as we show below. If we assemble particulars of the sisters of women (Index Cases) whose children have been affected by haemolytic disease of the newborn, then, since each Index Case is ipso facto Rh-negative, the sisters of Index Cases will include more Rhnegative individuals than a random sample of women. We may calculate the expected proportion of $\mathrm{Rh}$-negative individuals among sisters of Index Cases and thence the expected proportion of their children affected with haemolytic disease, as follows:

let $d=$ recessive gene frequency (of $d$-gene), so that $1-d=$ dominant gene frequency (of $D$-gene).

Recessive individuals may arise only from the three matings:

\begin{tabular}{c|c|c|c}
\hline $\begin{array}{c}\text { Type of } \\
\text { Mating }\end{array}$ & $\begin{array}{c}\text { Frequency of } \\
\text { Mating }\end{array}$ & $\begin{array}{c}\text { Proportion of } \\
\text { Recessive } \\
\text { Offspring }\end{array}$ & $\begin{array}{c}\text { Frequency of } \\
\text { Recessive } \\
\text { Offspring }\end{array}$ \\
\hline $\begin{array}{cc}\mathbf{R} \times \mathbf{R} \\
\text { B } \mathbf{H} \times \mathbf{R}\end{array}$ & $\begin{array}{c}d^{4} \\
4 d^{3}(1-d) \\
\text { C } \mathbf{H} \times \mathbf{H}\end{array}$ & \begin{tabular}{c}
$d^{2}(1-d)^{2}$ \\
\hline
\end{tabular} & $\begin{array}{c}d^{4} \\
2 d^{3}(1-d) \\
d^{2}(1-d)^{2}\end{array}$ \\
\hline
\end{tabular}

where $\mathbf{R}=$ Homozygous recessive; $\mathbf{H}=$ Heterozygote.
Thus a recessive individual results from the three mating groups (denoted above as $\mathrm{A}, \mathrm{B}, \mathrm{C}$ ) in the relative proportions $d^{2}: 2 d(1-d):(1-d)^{2}$. Sibs of such an individual will also be recessive in the same proportions as above, i.e. as $1: \frac{1}{2}: \frac{1}{4}$.

The total frequency of all recessive offspring of the matings from which recessive offspring may come is: $d^{4}+2 d^{3}(1-d)+d^{2}(1-d)^{2}=d^{2}$.

Hence the mean proportion of sibs of Index Cases (i.e. known recessives) expected also to be recessive is:

$$
d^{2}+d(1-d)+\frac{1}{4}(1-d)^{2}=\frac{1}{4}(1+d)^{2}
$$

wherein the assumptions we have made are that the Index Cases result from families A, B, C, in the randomly expected proportions, and that the size of a sibship bears no relation to whether it is of type $\mathrm{A}, \mathrm{B}$, or C.

Now those sisters of Index Cases who are recessive may marry Rh-positive men, when the circumstances would be propitious for the production of haemolytic disease. There will be a proportion $(1-d)^{2}$ of homozygous $\mathrm{Rh}$-positive males all of whose children will be Rh-positive, and a proportion $2 d(1-d)$ of heterozygous males, half of whose children by an Rh-negative woman will be Rh-positive. Thus the proportion of Rh-positive children to be expected if the Rh-negative sisters of Index Cases marry at random with respect to the $R h$ gene is:

$$
(1-d)^{2}+d(1-d)=(1-d)
$$

and the proportion of Rh-positive children of Rh-negative mothers which is expected among the children of sisters of Index Cases is:

$$
\frac{1}{4}(1+d)^{2}(1-d)
$$

if we may assume that the number of children per family is sensibly unaffected by their $\mathrm{Rh}$ genotype.

It has been observed that in general a proportion $p$ (about 5 per cent.) of Rh-positive children born to $\mathrm{Rh}$-negative mothers will show signs of icterus gravis, so that the proportion of the children of sisters of Index Cases expected to be affected is:

$$
\frac{1}{4} p(1-d)(1+d)^{2}
$$

If however, Wiener's hypothesis of an inherited factor of enhanced susceptibility is operative, we proceed as follows. 
If we assume that an Index Case possesses at least one dominant gene $K$, of frequency $x$, her parents' mating types may be:

\begin{tabular}{c|c|c|c}
\hline $\begin{array}{c}\text { Type of } \\
\text { Mating }\end{array}$ & $\begin{array}{c}\text { Frequency of } \\
\text { Mating }\end{array}$ & $\begin{array}{c}\text { Proportion of } \\
\text { Offspring of } \\
\text { Dominant } \\
\text { Phenotype }\end{array}$ & $\begin{array}{c}\text { Frequency of } \\
\text { Dominant } \\
\text { Offspring }\end{array}$ \\
\hline $\mathbf{R} \times \mathbf{H}$ & $4 x(1-x)^{3}$ & $\frac{1}{2}$ & $2 x(1-x)^{3}$ \\
$\mathbf{H} \times \mathbf{H}$ & $4 x^{2}(1-x)^{2}$ & 1 & $3 x^{2}(1-x)^{2}$ \\
$\mathbf{R} \times \mathbf{D}$ & $2 x^{2}(1-x)^{2}$ & 1 & $x^{2}(2-x)^{2}$ \\
$\mathbf{H} \times \mathbf{D}$ & $4 x^{3}(1-x)$ & 1 & \\
\hline
\end{tabular}

where $\mathbf{R}=$ Homozygous recessive $(k k)$

$\mathbf{H}=$ Heterozygote $(K k)$

$\mathrm{D}=$ Homozygous dominant $(K K)$

Again, the sibs of such an Index Case will also carry at least one dominant gene in the same proportions as above. Thus the frequency of pairs of sisters, each carrying a dominant gene, is obtained from the product of the last two columns, and is:

$$
p_{1}=\frac{1}{4} x\left(4+5 x-6 x^{2}+x^{3}\right) \text {. }
$$

The proportion of the general population who carry at least one dominant gene $K$ is:

$$
p_{2}=1-(1-x)^{2}=x(2-x),
$$

so that the probability, relative to the general population frequency, of a sister's carrying a gene is:

$$
\frac{p_{1}}{p_{2}}=\frac{1}{2}+\frac{7}{8} x,
$$

and this is the proportion of sisters of Index Cases who also carry the factor $K$.

The estimate of Wiener (1946) is 3 per cent. for heterozygous $K$ individuals, yielding approximately 0.015 for $x$, so that $\frac{p_{1}}{p_{2}}$ is about 51 per cent.

Thus of the proportion $\frac{1}{4}(1+d)^{2}(1-d)$ of susceptible offspring of sisters of Index Cases, those actually affected with haemolytic disease will be:

(i) approximately 5 per cent., if the chance is random,

(ii) approximately 51 per cent., on Wiener's postulate of a simple dominant gene $K$ conferring an enhanced susceptibility.

Thus Wiener's postulate leads to a proportion of affected which is about ten times greater than that expected in the absence of a dominant familial predisposition. This is of course on the assumption of independence of the $d$-gene and the $K$-gene. Linkage might increase the proportion by as much as about $\mathbf{4 0}$ per cent.

To test the merits of alternative hypotheses, we have made use of the cases of icterus gravis treated at the Children's Hospital, Birmingham, between 1920 and 1947, recently reviewed by one of us (Gerrard, 1951). Because the likelihood of occurrence of iso-immunization is reduced in small families, and to allow of the inclusion of a greater proportion of younger sisters' families, we have drawn chiefly from the older mothers in the series. Through them we have obtained details of the families of their sisters, the numbers of children, and the numbers who were deeply jaundiced in infancy. We obtained the required details from 111 Index Cases of the series, and in five instances one sister, in a sixth, two sisters, had had children who were severely jaundiced as infants. The 111 Index Cases had a total of 274 sisters who had 566 children. Eleven of these children were severely jaundiced in infancy, and in at least nine of these the jaundice was due to $\mathrm{Rh}$ iso-immunization.

The expected number of cases is $8 \cdot 3$ calculated according to (i), and 85.4 according to (ii). This series therefore lends no support to the postulate of a genetic factor facilitating sensitization such as Wiener has proposed; indeed the close accord between the number observed and that expected in the absence of the factor $K$ suggests that the greater susceptibility to sensitization which some $\mathrm{Rh}$-negative women display is distributed independently and is attributable to some environmental agency. For any other condition facilitating isoimmunization, whether directly improving antigenic response or increasing the permeability of the placenta, if dependent on a single gene substitution, would increase the proportion affected among the offspring of sisters from the 5 per cent. rate of $(i)$ to a minimum of 25 per cent., as a recessive with full penetrance.

\section{SUMMARY}

Observations on the occurrence of haemolytic disease of the newborn in the offspring of sisters are used to show, by their close agreement with genetic expectation on the assumption of random distribution of susceptibility, that any hypothesis of a simply-inherited factor which facilitates sensitization is improbable.

\section{REFERENCES}

Gerrard, J. W. (1951). "The Natural History of Kernicterus." Dissertation for degree of D.M. (Oxon.).

Wiener, A. S. (1946). Amer. J. Dis. Child., 71, 14. 